\title{
A model for predicting post-liver transplantation recurrence in intrahepatic cholangiocarcinoma recipients
}

\author{
Ao Ren ${ }^{1,2,3}$, Zhongqiu Li ${ }^{1,2,3}$, Xuzhi Zhang ${ }^{1,2,3}$, Ronghai Deng $^{1,2,3}$, Yi Ma ${ }^{1,2,3}$ \\ ${ }^{1}$ Organ Transplant Center, The First Affiliated Hospital, Sun Yat-sen University, Guangzhou, China; ${ }^{2}$ Guangdong Provincial Key Laboratory of \\ Organ Donation and Transplant Immunology, The First Affiliated Hospital, Sun Yat-sen University, Guangzhou, China; ${ }^{3}$ Guangdong Provincial \\ International Cooperation Base of Science and Technology (Organ Transplantation), The First Affiliated Hospital, Sun Yat-sen University, \\ Guangzhou, China \\ Contributions: (I) Conception and design: A Ren, Y Ma; (II) Administrative support: Y Ma; (III) Provision of study materials or patients: A Ren, Z \\ Li; (IV) Collection and assembly of data: Z Li, X Zhang; (V) Data analysis and interpretation: A Ren, Z Li, X Zhang; (VI) Manuscript writing: All \\ authors; (VII) Final approval of manuscript: All authors. \\ Correspondence to: Dr. Yi Ma. Organ Transplant Center, the First Affiliated Hospital, Sun Yat-sen University, No.58 Zhongshan Er Road, Guangzhou \\ 510080, China. Email: anhuimayi2002@163.com.
}

\begin{abstract}
Background: It is important to select appropriate patients for improving the outcomes of liver transplantation for intrahepatic cholangiocarcinoma (iCCA). The objective of this study was to establish a predictive model for the recurrence of iCCA after liver transplantation.

Methods: Twenty-one patients who received liver transplantation for iCCA were used to construct a model for predicting recurrence. Predictors of recurrence were tested by a Cox model analysis. The results were validated in 28 patients who were followed up.

Results: Diameter and number of tumors, and CA19-9 level independently predicted tumor recurrence. At a Cox score threshold of 0.736 [95\% confidence interval (CI): 0.549-0.923], a model combining these factors was highly predictive of tumor recurrence and death. A simplified version of the model identified a cut-off value of 6 . The 5 -year OS rate was lower in patients with a score $>6$ points compared to those with a score $\leq 6$ points $(\mathrm{P}=0.005)$. The 5 -year recurrence rate was higher in patients with a score $>6$ points as compared to those with a score $\leq 6$ points $(\mathrm{P}=0.034)$.
\end{abstract}

Conclusions: The model developed was useful for predicting recurrence of iCCA after liver transplantation. Large sample, multicenter studies are needed to validate the findings.

Keywords: Intrahepatic cholangiocarcinoma; liver transplantation; model

Submitted May 07, 2020. Accepted for publication Sep 20, 2020.

doi: 10.21037/jgo-20-209

View this article at: http://dx.doi.org/10.21037/jgo-20-209

\section{Introduction}

Cholangiocarcinoma (CCA) is classified into intrahepatic (iCCA), perihilar, and distal subtypes, which accounts for $8-10 \%$ of biliary tract cancers $(1,2)$. The incidence of iCCA has been increasing over the last 3 decades. While the exact reason of the increased incidence is controversial, which may be related to primary sclerosing cholangitis, viral hepatitis, or chemical exposure (3). Surgery offers the only opportunity for long-term survival, but 5 -year survival after curative intent resection remains at only $20-30 \%$ (4).
However, most iCCA tumors are considered to be unresectable at the time of diagnosis because of the lack of early clinical symptoms. Thus, liver transplantation is the remaining promising treatment for iCCA. However, data from several studies have reported poor post-transplant outcomes in patients with iCCA $(5,6)$. Thus, iCCA is consider to be a contraindication to liver transplantation (7). On the other hand, recent data have shown excellent outcomes in selected patients, thereby justifying transplantation for iCCA patients under restrictive 
conditions $(8,9)$. Current criteria for evaluating liver transplantation, such as the Milan criteria, that are effective for hepatocellular carcinoma (HCC) patients are not useful for evaluating patients with iCCA. Currently, there is no sufficient criterion for evaluating liver transplantation in patients with iCCA.

In the last few years, some studies have indicated that tumor diameter $>2 \mathrm{~cm}$ and multinodular are risk factors for recurrence and poorer 5 -year survival in patients with iCCA $(10,11)$. In these studies, a single tumor and size $\leq 2 \mathrm{~cm}$ was defined as "very early" iCCA, and all other cases were defined as "advanced" iCCA. Patients with early iCCA have lower recurrence rate and higher survival rate after liver transplantation compared with patients with advanced iCCA. These data support that early iCCA patients should be considered as candidates for liver transplantation. Other study has shown that high CA19-9 levels are predictive of a poor postoperative prognosis (12).

Thus, the objectives of this study were to establish and validate a predictive model for the recurrence and prognosis of iCCA after liver transplantation incorporating CA19-9, tumor size, and tumor number. We present the following study in accordance with the STROBE reporting checklist (available at http://dx.doi.org/10.21037/jgo-20-209).

\section{Methods}

The study population consisted of patients who had undergone liver transplantation for iCCA at The First Affiliated Hospital, Sun Yat-sen University between 2008 and 2017. This study only included patients with iCCA at the explant. Patients with mixed hepatocellular cholangiocarcinoma tumors, transplant recipients who were $<18$ years old, and those without complete clinical data were excluded from the study. Twenty-seven patients were excluded due to the exclusion criteria. Twenty-one patients were used to develop the model, and 28 patients were used to validate the model. The patients in the discovery cohort were from 2008 to 2012, and the patients in the validation cohort were from 2012 to 2017.

Demographics, model for end-stage liver disease (MELD) score, cause of cirrhosis, liver function, CA199 values, imaging tumor features, pathologic diagnosis and type of pretransplant bridging therapies were retrospectively collected. The follow-up data included iCCA recurrence or death, cause of death and date of last follow-up.

All patients with iCCA on the waiting list were excluded from extrahepatic metastasis by imaging examination pre LT. Patients with a waiting time of more than 6 months could be treated with ablation and transarterial chemoembolization (TACE) as pretransplant bridging therapies. In addition to TACE and ablation, patients with iCCA diagnosed preoperatively received chemotherapy based on gemcitabine and cisplatin.

Eight potential predictors were screened to determine which variables were independent predictors of recurrence, which were selected based on clinical experience and previous studies: age, gender, MELD scores, HBV-DNA, number of tumor, CA19-9 level, tumor size, preoperative treatment. Univariate and multivariate analysis were performed to evaluate the potential value of risk factors.

\section{Statistical analysis}

Multivariate analysis using the cox proportional hazards regression model was used to determine the risks for post-LT recurrence. A cut-off value was derived from the area under the receiver operating characteristic (ROC) curve(AUC) based on the highest Youden index. The predictive model was examined in the validation cohort and assessed for calibration using the Hosmer-Lemeshow C test. Survival curves were generated with the Kaplan-Meier method, and compared with the log-rank test. All data were analyzed using SPSS version 19.0 (SPSS, Chicago, IL, USA). Statistical significance was considered as $\mathrm{P}<0.05$.

All organs came from voluntary donations from citizens; no organs from executed prisoners (even with his/her consent) were used involved. Because of the retrospective nature, the requirement of informed consent was waived. The study was approved by the Institutional Review Board of the The First Affiliated Hospital, Sun Yat-sen University [Approval No. (2020) 344] and was performed in accordance with the Declaration of Istanbul. All protocols conformed to the ethical guidelines of the 1975 Helsinki Declaration (as revised in 2013).

\section{Results}

Table 1 shows the demographic and clinical characteristics of 49 consecutive adult liver transplant patients with iCCA who met the inclusion criteria. All patients diagnosed with iCCA received adjuvant therapy Gemcitabine and Cisplatin, part of patients received ablation and TACE as pretransplant bridging therapies.

Of the 8 potential risk factors for recurrence, CA19-9 level, largest tumor diameter, and number of nodules were 
Table 1 Demographics of patients in the study group

\begin{tabular}{|c|c|c|c|}
\hline Demographics & Validation cohort $(n=28)$ & Discovery cohort $(n=21)$ & $\mathrm{P}$ \\
\hline $\operatorname{Sex}(M), n(\%)$ & $25(89.29)$ & $19[90.48]$ & 0.892 \\
\hline Cause of cirrhosis, $n(\%)$ & & & 0.802 \\
\hline HBV & $13(46.43)$ & $9(42.86)$ & \\
\hline Alcoholic disease & $0(0)$ & $0(0)$ & \\
\hline Median MELD score (IQR) & 7 [2-34] & $6[4-18]$ & 0.742 \\
\hline Number of nodules, n (\%) & & & 0.441 \\
\hline Uninodular & $19(67.86)$ & $12(57.14)$ & \\
\hline Median CA19-9 value at diagnosis (IQR), $\mathrm{ng} / \mathrm{mL}$ & $383.15[2-20,000]$ & $307.4[5.8-12,000]$ & 0.613 \\
\hline Types of tumor treatment, $\mathrm{n}(\%)$ & & & 0.037 \\
\hline TACE & $12(42.86)$ & $6(28.57)$ & \\
\hline Ablation & $6(21.43)$ & $2(9.52)$ & \\
\hline TACE + ablation & $3(10.71)$ & $1(4.76)$ & \\
\hline Tumor differentiation, $\mathrm{n}(\%)$ & & & 0.493 \\
\hline Well-differentiated & $2(7.14)$ & $2(9.52)$ & \\
\hline Mod-differentiated & $18(64.29)$ & $16(76.19)$ & \\
\hline Both hepatic and extrahepatic & $6(21.43)$ & $3(14.29)$ & \\
\hline Median time on waiting list (IQR), months & $4.25[2.75-5.78]$ & $4.5[3-6.9]$ & 0.663 \\
\hline Median follow-up (IQR), months & $20.5[14.5-42.25]$ & 27 [17-43] & 0.334 \\
\hline
\end{tabular}

significantly associated with tumor recurrence (Table 2).

The $\beta$ regression coefficient values and hazard ratios (HRs) for each independent predictor of iCCA recurrence are summarized in Table 3. The AUC of the model for predicting recurrence was 0.736 [95\% confidence interval (CI): $0.549-0.923$ ], with a sensitivity of $69.2 \%$ and a specificity of $73.3 \%$.

A simplified version of the model is presented in Table 4. The simplified version was based on the original model by linear transformation of the $\beta$ coefficients ( $\beta$ coefficients $\times 3$, rounded). In the simplified version scores were calculated by adding the individual values of each variable, and a cutoff value of 6 separated patients with high- or low-risk of recurrence (low-risk patients score $\leq 6$, high-risk patients score $>6$ ). The selection of the cut-off value was based on the cut-off value of the Cox score. The selection of the cutoff value was based on the cut-off value of Cox score 0.736 .

The overall survival (OS) and recurrence rates according 
Table 2 Univariate analysis of preoperative factors affecting tumor recurrence in the discovery cohort

\begin{tabular}{lcc}
\hline Category & Data & Univariate analysis \\
\hline Gender, $\mathrm{n}(\%)$ & & 0.476 \\
Male & $19(90.48)$ & \\
Female & $2(9.52)$ & \\
Median age (IQR), years & $55[33-67]$ & 0.818 \\
HBV-DNA, n (\%) & & 0.717 \\
Positive & $9(42.86)$ & \\
Negative & $12(57.14)$ & \\
Median MELD score (IQR) & $6[4-18]$ & 0.546 \\
Number of tumor nodules, $\mathrm{n}(\%)$ & & 0.021 \\
Uninodular & $12(57.14)$ & \\
Multinodular & $9(42.86)$ & \\
CA19-9 level, ng/mL, $\mathrm{n}(\%)$ & & 0.016 \\
$\leq 100$ & & \\
$100-1,000$ & $5(23.80)$ & \\
$>1,000$ & $8(38.10)$ & \\
Diameter of the largest tumor, cm, $\mathrm{n}(\%)$ & \\
$\leq 2$ & $7(33.33)$ & \\
2-5 & & \\
$>5$ & & \\
TACE + ablation & & \\
\hline
\end{tabular}

to the simplified cut-off value of 6 were examined in the development cohort and the validation cohort. In the development cohort, the 1-, 3-, and 5-year recurrence rates were higher in patients with a score $>6$ points as compared to those with a score $\leq 6$ points $(44.44 \%, 70.37 \%$, and $70.37 \%$ vs. $18.18 \%, 27.27 \%$, and $27.27 \%$, respectively, $\mathrm{P}=0.03$ ). In addition, the 1-, 3-, and 5-year OS rates were lower in patients with a score $>6$ points compared to those with a score $\leq 6$ points $(80 \%, 20 \%$, and $20 \%$ vs. $100 \%$, $72.73 \%$, and $60.61 \%$, respectively, $\mathrm{P}=0.034$ ) (Figure 1 ).

Similar results were obtained in the validation cohort. The 1-, 3-, and 5-year recurrence rates were higher in patients with a score $>6$ points compared to those with a score $\leq 6$ points $(38.89 \%, 66.05 \%$, and $66.05 \%$ vs. $11.11 \%$,
Table 3 Independent predictors of tumor recurrence after transplantation in the discovery cohort based on multivariate analysis $(\mathrm{n}=21)$

\begin{tabular}{lcccc}
\hline Variables & $\beta$ coefficient & $\mathrm{HR}$ & $95 \% \mathrm{Cl}$ & $\mathrm{P}$ \\
\hline $\begin{array}{l}\text { Largest diameter } \\
\text { <2 cm }\end{array}$ & 0 & 1 & & \\
$2-5 \mathrm{~cm}$ & 1.096 & 2.994 & $1.098-8.160$ & 0.032 \\
$>5 \mathrm{~cm}$ & 1.340 & 3.820 & $1.494-9.767$ & 0.005 \\
Number of nodules & & & & \\
1 & 0 & 1 & & \\
$2-3$ & 0.513 & 1.670 & $0.874-3.193$ & 0.121 \\
$\geq 4$ & 1.625 & 5.081 & $2.468-10.460$ & 0.000 \\
CA19-9 level (ng/mL) & & & & \\
$\leq 100$ & 0 & 1 & & \\
$100-1,000$ & 0.298 & 1.348 & $0.602-3.017$ & 0.468 \\
$>1,000$ & 0.914 & 2.493 & $1.174-5.296$ & 0.017 \\
\hline
\end{tabular}

Table 4 Simplified version of this model

\begin{tabular}{lccc}
\hline Variables & $\beta$ coefficient & HR & Points \\
\hline Largest diameter & 0 & 1 & \\
$\leq 2 \mathrm{~cm}$ & 1.096 & 2.994 & 3 \\
$2-5 \mathrm{~cm}$ & 1.340 & 3.820 & 4 \\
$>5 \mathrm{~cm}$ & & & \\
Number of nodules & 0 & 1 & \\
1 & 0.513 & 1.670 & 2 \\
$2-3$ & 1.625 & 5.081 & 5 \\
$\geq 4$ & & & \\
CA19-9 level (ng/mL) & 0 & 1 & \\
$\leq 100$ & 0.298 & 1.348 & 1 \\
$100-1,000$ & 0.914 & 2.493 & 3 \\
$>1,000$ & & & \\
\hline
\end{tabular}

Scores are calculated by adding the individual score for each variable. The cut-off value of 6 selected exactly according to the Cox score cut-off value of 0.736 .

$22.22 \%$, and $22.22 \%$, respectively, $\mathrm{P}=0.034)$. The $1-, 3-$, and 5 -year OS rates were lower in patients with a score $>6$ points compared to those with a score $\leq 6$ points $(78.95 \%$, $26.31 \%$, and $15.79 \%$ vs. $88.89 \%, 66.67 \%$, and $33.33 \%$, respectively, $\mathrm{P}=0.005$ ) (Figure 2). 

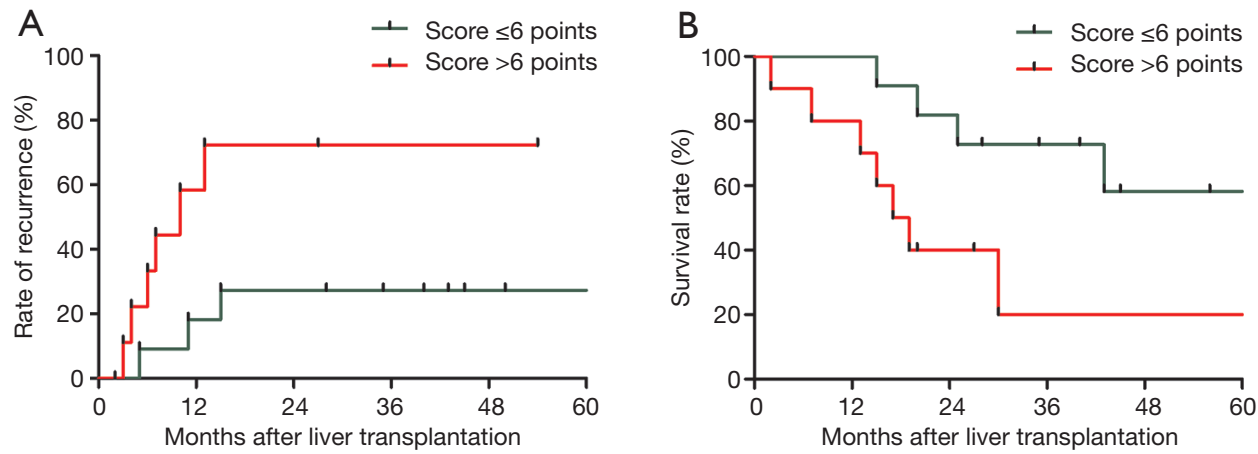

Figure 1 Risk of recurrence (A) and overall survival (B) according to the score cut off of 6 in discovery cohort.
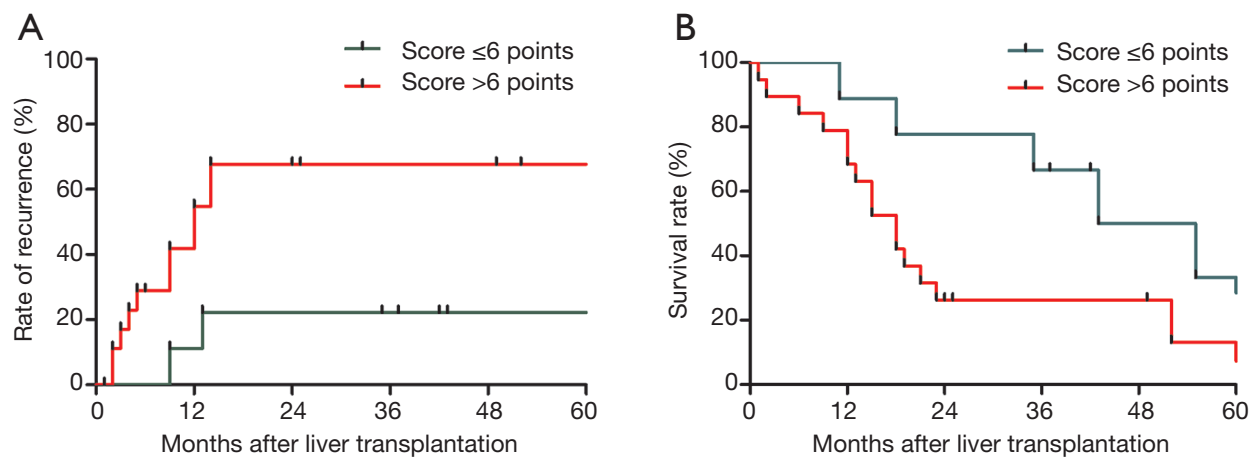

Figure 2 Risk of recurrence (A) and overall survival (B) according to the score cut off of 6 in validation cohort.

\section{Discussion}

Compared with HCC, iCCA has a higher recurrence rate and a worse prognosis. In 2014, Sapisochin et al. retrospectively analyzed 44 patients with HCC or iCCA (27 had an iCCA), and found that patients with iCCA had a higher 5 -year recurrence rate than HCC $(36 \%$ vs. $2 \%$ ), and lower 5 -year OS (51\% vs. 93\%) (13). As such, liver transplantation for iCCA is highly controversial and because of the poor long-term survival and high recurrence rates liver transplantation for iCCA has been abandoned in most transplantation centers.

While the indications for liver transplantation for iCCA are controversial, several studies have shown that iCCA patients with small solitary tumors have good long-term survival after liver transplantation. In 2016, Sapisochin et al. (10) conducted a multicenter study that included 48 patients with iCCA. Among them, 15 patients had very early iCCA and 33 patients had advanced disease (single tumor $>2 \mathrm{~cm}$ or multifocal disease). The advanced group had a higher 5 -year recurrence rate than the very early iCCA group $(61 \%$ vs. $15 \%$, respectively), and lower 5 -year OS ( $45 \%$ vs. $65 \%$, respectively). Therefore, appropriate selection criteria are required to ensure a better prognosis of patients undergoing liver transplantation for iCCA.

In addition to the Milan criteria for evaluating patients with HCC, several other selection criteria have been proposed and include the Up-to-7 criteria, Hangzhou criteria, the AFP French model, and UCSF criteria (14). However, is no specific criterion for evaluating iCCA patients for liver transplantation. We explore the establishment of specific criteria for iCCA in order to facilitate the selection of appropriate patients to ensure better outcomes after liver transplantation.

The size and number of tumors play an important role in the prognosis of patients undergoing LT for iCCA. In 2014, a multicentre study demonstrated that liver transplantation for patients with very early iCCA (single tumor $\leq 2 \mathrm{~cm}$ ) could achieve a 5 -year survival rate of $73 \%(11,13)$. A multicenter study performed at 17 large institutions worldwide also confirmed that patients with very early iCCA have an acceptable 5-year survival rate 
and a low recurrence rate after liver transplantation (10). Studies have explored the significance of CA19-9 in iCCA, and reported it is associated with recurrence and decreased long-term survival after resection $(15,16)$. Some studies have included CA19-9 level in iCCA staging, and a recent study reported that staging system for predicting survival of iCCA patients after LT, which classified patients with iCCA into four prognostic stages $(17,18)$. Another study showed that a preoperative CA19-9 serum level of $<100 \mathrm{U} / \mathrm{mL}$ was associated with better outcomes in patients with iCCA who received macroscopic curative resection (19). In the present study, we showed that size and number of tumors, and serum CA19-9 level were independent predictors of recurrence after transplantation for iCCA, and also predicted post-transplantation survival.

Other models have been proposed for predicting the prognosis of iCCA after hepatectomy, and are based on alkaline phosphatase, CA 19-9, CEA, number of tumors, tumor size, and tumor boundary type $(20,21)$. On this basis, we designed and validated a new predictive model that combined CA19-9 serum level and tumor size and number. Although the model was based on the analysis of only 28 patients, it exhibited good predictive value for iCCA recurrence after LT. A simplified version of the model was developed for predicting recurrence of iCCA based on the highest Youden index of ROC curve, which was 6. A score was calculated for each patient based on the simplified version of the model. Patients with a model score $\leq 6$ had significantly better outcome than patients with a score $>6$. Only $30-40 \%$ of patients with iCCA were deemed to benefit from surgical resection $(7,22)$, and 5 -year OS rate of patients with iCCA undergoing surgical resection varies between $11 \%$ and $40 \%(20,23,24)$. As for LT, in a multicenter study from Spain, the 5 -year survival rate of LT for patients with very early iCCA could be $73 \%$ (8). Collectively, these data suggest that select suitable iCCA patients for liver transplantation to achieve better prognosis. As for locoregional treatments and systematic therapy, TACE and ablation are locoregional treatment options that could prolong survival in iCCA patients, especially recommended for the local control of small and localized lesions without extrahepatic spread (25). The role of systematic therapy in patients with unresectable iCCA is more clearly demonstrated, and proved the benefit in patients underwent $\mathrm{R} 1$ resection and with $\mathrm{LN}$ metastasis (26).

This study has several limitations. This was a retrospective, single-center analysis with a small number of patients, and with a long inclusion period, which might limit the reproducibility of the results. However, it is important to consider the rarity of iCCA, and the fact that fewer patients receive liver transplantation for iCCA than hepatectomy.

In conclusion, we identified 3 independent risk factors for recurrence in iCCA patients post LT, and set up a model based on these preoperative factors to improve the selection of iCCA patients at low risk of recurrence post LT. Based on the model, we propose combining CA19-9, tumor number, and tumor size to select suitable iCCA patients for liver transplantation. The simplified version of the model was also established to facilitate clinical application. However, given the limitations mentioned above, this model needs further validation and improvement with a larger multicentre sample of more defined subjects.

\section{Acknowledgments}

Funding: This work was supported by grants from the National Natural Science Foundation of China, China (81873591, and 81670591), Guangdong Natural Science Foundation, China (2016A030311028), The Science and Technology Planning Project of Guangdong Province, China (2018A050506030), and Science and Technology Program of Guangzhou, China (201704020073). The Guangdong Provincial Key Laboratory Construction Projection on Organ Donation and Transplant Immunology (2013A061401007 and 2017B030314018), Guangdong Provincial International Cooperation Base of Science and Technology (Organ Transplantation) (2015B050501002).

\section{Footnote}

Reporting Checklist: The authors have completed the STROBE reporting checklist. Available at http://dx.doi. org/10.21037/jgo-20-209

Data Sharing Statement: Available at http://dx.doi. org/10.21037/jgo-20-209

Peer Review File: Available at http://dx.doi.org/10.21037/ jgo-20-209

Conflicts of Interest: All authors have completed the ICMJE uniform disclosure form (available at: http://dx.doi. org/10.21037/jgo-20-209). The authors have no conflicts of interest to declare. 
Ethical Statement: The authors are accountable for all aspects of the work in ensuring that questions related to the accuracy or integrity of any part of the work are appropriately investigated and resolved. Because of the retrospective nature, the requirement of informed consent was waived. The study was approved by the Institutional Review Board of the The First Affiliated Hospital, Sun Yat-sen University [Approval No. (2020) 344] and was performed in accordance with the Declaration of Istanbul. All protocols conformed to the ethical guidelines of the 1975 Helsinki Declaration (as revised in 2013).

Open Access Statement: This is an Open Access article distributed in accordance with the Creative Commons Attribution-NonCommercial-NoDerivs 4.0 International License (CC BY-NC-ND 4.0), which permits the noncommercial replication and distribution of the article with the strict proviso that no changes or edits are made and the original work is properly cited (including links to both the formal publication through the relevant DOI and the license). See: https://creativecommons.org/licenses/by-nc-nd/4.0/.

\section{References}

1. Bergquist A, Von SE. Epidemiology of cholangiocarcinoma. Best Pract Res Clin Gastroenterol 2015;29:221-32.

2. Gupta A, Dixon E. Epidemiology and risk factors: intrahepatic cholangiocarcinoma. Hepatobiliary Surg Nutr 2017;6:101.

3. Vogel A, Saborowski A. Cholangiocellular Carcinoma. Digestion 2017;95:181.

4. Mavros MN, Economopoulos KP, Alexiou VG, et al. Treatment and Prognosis for Patients With Intrahepatic Cholangiocarcinoma. JAMA Surg 2014;149:565-74.

5. Meyer CG, Penn I, James L. Liver Transplantation For Cholangiocarcinoma: Results In 207 Patients1. Transplantation 2000;69:1633-7.

6. Becker NS, Rodriguez JA, Barshes NR, et al. Outcomes analysis for 280 patients with cholangiocarcinoma treated with liver transplantation over an 18 -year period. J Gastrointest Surg 2008;12:117-22.

7. Bridgewater J, Galle PR, Khan SA, et al. Guidelines for the diagnosis and management of intrahepatic cholangiocarcinoma. J Hepatol 2014,60:1268-89.

8. Sapisochin G, Rodríguez de Lope C, Gastaca M, et al. "Very early" intrahepatic cholangiocarcinoma in cirrhotic patients: should liver transplantation be reconsidered in these patients? Am J Transplant 2014;14:660-7.

9. Goldaracena N, Gorgen A, Sapisochin G. Current Status of Liver Transplantation for Cholangiocarcinoma. Liver Transpl 2018;24:294-303.

10. Sapisochin G, Facciuto M, Rubbia Brandt L, et al. Liver transplantation for "very early" intrahepatic cholangiocarcinoma: International retrospective study supporting a prospective assessment. Hepatology 2016;64:1178-88.

11. Hashimoto K, Miller CM. Liver transplantation for intrahepatic cholangiocarcinoma. J Hepatobiliary Pancreat Sci 2015;22:138-143.

12. Zheng BH, Yang LX, Sun QM, et al. A New Preoperative Prognostic System Combining CRP and CA199 For Patients with Intrahepatic Cholangiocarcinoma. Clin Transl Gastroenterol 2017;8:e118.

13. Sapisochin G, Lope CRD, Gastaca M, et al. Intrahepatic Cholangiocarcinoma or Mixed Hepatocellular-

Cholangiocarcinoma in Patients Undergoing Liver Transplantation: A Spanish Matched Cohort Multicenter Study. Ann Surg 2014;259:944-52.

14. Burra P, Zanetto A, Rodriguez-Castro KI. HCC criteria for liver transplantation: Controversies. Liver Transpl 2015;21:S27.

15. Kondo N, Murakami Y, Uemura K, et al. Elevated perioperative serum CA 19-9 levels are independent predictors of poor survival in patients with resectable cholangiocarcinoma. J Surg Oncol 2014;110:422-9.

16. Chung MJ, Lee KJ, Bang S, et al. Preoperative Serum CA 19-9 Level as a Predictive Factor for Recurrence after Curative Resection in Biliary Tract Cancer. Ann Surg Oncol 2011;18:1651-6.

17. Chaiteerakij R, Harmsen WS, Marrero CR, et al. A New Clinically Based Staging System for Perihilar Cholangiocarcinoma. Am J Gastroenterol2014;109:1881-90.

18. Zhou H, Jiang X, Li Q, et al. A simple and effective prognostic staging system based on clinicopathologic features of intrahepatic cholangiocarcinoma. Am J Cancer Res 2015;5:1831-43.

19. Huang J, Wang $X$, Zhu $Y$, et al. Specific prognostic factors in hepatitis B virus related and non hepatitis B virusrelated intrahepatic cholangiocarcinoma after macroscopic curative resection. J Surg Oncol 2019;119:40-6.

20. Wang Y, Li J, Xia Y, et al. Prognostic Nomogram for Intrahepatic Cholangiocarcinoma After Partial Hepatectomy. J Clin Oncol 2013;31:1188-95.

21. Jiang $\mathrm{W}$, Zeng ZC, Tang ZY, et al. A prognostic 
scoring system based on clinical features of intrahepatic cholangiocarcinoma: The Fudan score. Ann Oncol 2011;22:1644-52.

22. Weber SM, Ribero D, O'Reilly EM, et al. Intrahepatic cholangiocarcinoma: expert consensus statement. HPB 2015;17:669-80.

23. Nakagohri T, Kinoshita T, Konishi M, et al. Surgical outcome and prognostic factors in intrahepatic cholangiocarcinoma. World J Surg 2008;32:2675-80.

24. Yoo T, Park SJ, Han SS, et al. Postoperative CA19-

Cite this article as: Ren A, Li Z, Zhang X, Deng R, Ma Y. A model for predicting post-liver transplantation recurrence in intrahepatic cholangiocarcinoma recipients. J Gastrointest Oncol 2020;11(6):1283-1290. doi: 10.21037/jgo-20-209
9 Change Is a Useful Predictor of Intrahepatic Cholangiocarcinoma Survival following Liver Resection. Dis Markers 2015;2015:298985.

25. Kim JH, Won HJ, Shin YM, et al. Radiofrequency Ablation for the Treatment of Primary Intrahepatic Cholangiocarcinoma. AJR Am J Roentgenol 2011;196:W205-9.

26. Valle J, Wasan H, Palmer DH, et al. Investigators, Cisplatin plus gemcitabine versus gemcitabine for biliary tract cancer. N Engl J Med 2010;362:1273-81. 